\title{
Proyecto Nexos. Una experiencia de articulación entre la escuela secundaria y la Universidad Nacional del Litoral
}

Palabras clave. inclusión educativa · articulación de niveles $\cdot$ tutorías $\cdot$ docentes

(1) Profesora y Licenciada en Gestión Educativa. Directora de Articulación e Ingreso de la Universidad Nacional del Litoral. Docente ordinaria de la Cátedra Política Educativa y Organización Escolar, y a cargo de las cátedras: Política y Legislación Educativa y Organización de las Instituciones

Educativas, que se ofrecen para los Ciclos de Licenciatura en Gestión Educativa (PROCAT) marcelblesio@gmail.com

(2) Estudiante de la Licenciatura en Ciencias Política de la Facultad de Humanidades y Ciencias. Becario del Área de Articulación e Ingreso de la Universidad Nacional del Litoral.
(3) Licenciada en Sociología. Becaria del Área de Articulación e Ingreso de la Universidad Nacional del Litoral. Docente de la Cátedra Seminario de Tesina de la Carrera de Lic. en Trabajo Social, de la Facultad de Ciencias Jurídicas y Sociales de la UNL. Docente JTP de las cátedras sociología y epistemología de la Carrera de Kinesiología de la Universidad del Gran Rosario.

(4) Licenciada en Biodiversidad. Becaria Doctoral coNICET. Coordinadora del Programa de Ingreso y Articulación de Niveles de la Universidad

Nacional del Litoral. Integrante de la Cátedra Química

General e Inorgánica de la

Facultad de Humanidades y Ciencias, UNL.
MARCEL MÓNICA BLESIO ${ }^{(1)}$

RODRIGO FACUNDO HERNÁNDEZ (2)

IVANA SOLEDAD MORELLI (3)

LUCÍA MAGDALENA ODETTI ${ }^{(4)}$

Fecha de recepción 29/04/19

Fecha de aceptación: 30/09/19

Resumen. El presente artículo reflexiona sobre una experiencia de articulación de niveles llevada a cabo en el marco del Proyecto Nexos, aprobado por la Secretaría de Políticas Universitarias del Ministerio de Educación y Deportes de la Nación, e implementado por la Dirección de Articulación e Ingreso, dependiente de la Secretaría de Planeamiento Institucional y Académico de la Universidad Nacional del Litoral; y el Ministerio de Educación de la Provincia de Santa Fe. El trabajo tuvo como principales destinatarios a las escuelas secundarias de la región, y especialmente a las de la provincia de Santa Fe. En este sentido, daremos cuenta del desarrollo de diferentes acciones implementadas durante los meses de mayo a diciembre de 2018, que promovieron la articulación entre la Educación Superior Universitaria y las escuelas secundarias, con el objetivo de fomentar y favorecer el ingreso a la universidad de los jóvenes. Asimismo, se buscó generar condiciones institucionales y curriculares que posibiliten procesos genuinos de inclusión y permanencia en los estudios universitarios, así como también la continuidad de estudios superiores a través del trabajo articulado entre docentes y estudiantes de ambos niveles. 


\section{'Nexos' project. An articulating instance between high school and Universidad Nacional del Litoral}

\begin{abstract}
This article reflects on an experience of articulation of levels, which was developed within the framework of a Project called Nexos, which was approved by the University Policies Department of the Argentine Ministry of Education and Sports and implemented by Articulation and Admission Office, which depends on the Department of Institutional and Academic Planning of Universidad Nacional del Litoral; and the Provincial Ministry of Education of Santa Fe. The project was intended for schools located in the region, in particular those in the Province of Santa Fe. In this sense, this paper accounts for different actions taken from May to December 2018, promoting the articulation
\end{abstract}

between university education and secondary schools, thus encouraging and favoring the access of youth to university studies. Also, it was intended to generate institutional and curricular conditions which enable genuine inclusive and permanence processes regarding both university studies and the continuity of higher studies through collaborative work among teachers and students at both levels.

Keywords. postgraduate experience . interinstitutional actions · training for school management

\section{Introducción}

La Conferencia Regional de Educación Superior celebrada en la Universidad Nacional del Córdoba (UNC) en 2018, en el marco de los cien años de la Reforma Universitaria, ratifica en sus principios declarativos a la educación superior como un bien público socia -estratégico; un deber del Estado, un espacio del conocimiento, derecho humano y universal; e indica que su ejercicio profundiza la democracia y posibilita la superación de las inequidades. En ella se despliega el compromiso con el ser humano, con su contexto, con el desarrollo en valores e ideales capaces de inspirar una cultura de paz, con respeto por los derechos humanos en un ambiente de garantías democráticas en el contexto de los objetivos de desarrollo sostenible.

En el contexto de democratización del sistema de educación superior característico de las sociedades contemporáneas, un abanico de problemáticas emergentes ha guiado, en las últimas décadas, la construcción de agendas de investigación y los debates sobre política universitaria. Nuestra Universidad no 
ha sido ajena a estas discusiones y hace más de dos décadas la problemática del acceso, la permanencia y la desafiliación durante los primeros años de la vida universitaria; la incorporación de sujetos con rasgos heterogéneos; la relación entre los conocimientos y las habilidades promovidas en los trayectos universitarios y la posterior inserción al mundo del trabajo, han formado parte de la agenda de las diferentes gestiones.

En este sentido, los marcos normativos -Estatuto (2012) y Plan de Desarrollo Institucional "Hacia la Universidad del Centenario» (2010)- constituyen un precedente en relación a dichas temáticas, asociadas a promover la democratización de la educación superior y generar mejores condiciones institucionales para el ingreso, la permanencia y el egreso de los estudios superiores. Durante las últimas décadas, se desarrolló un proyecto denominado: «Articulación interinstitucional para una mayor inclusión educativa. Políticas de articulación, acceso y acompañamiento a los estudiantes en los ciclos iniciales de las carreras de la UNL» en el que convergen tres grandes acciones para su abordaje: 1) actividades que comprenden espacios para la difusión de la propuesta académica de la universidad y de orientación educativa sobre la base del intercambio entre estudiantes de ambos niveles educativo; 2) el ofrecimiento de Cursos de Articulación -disciplinares y generales- como ámbito inicial obligatorio y no eliminatorio de inserción en los estudios universitarios; y 3) el apoyo a la permanencia de los estudiantes en los primeros años a través de becas a tutores pares que acompañan el proceso de inicio del cursado, brindando orientación institucional y académica.

\section{Algunas reflexiones sobre la situación actual}

En sus orígenes, los diferentes niveles del sistema educativo fueron concebidos con lógicas propias e independientes, lo que hizo de la articulación una cuestión fundamental. Fortalecerla implica, entonces, superar la idea de discontinuidad entre niveles en pos de una visión del sistema educativo obligatorio como un todo articulado, dentro del cual los mismos constituyen «etapas» de un proceso continuo de aprendizaje. Esto supone necesariamente promover la coherencia 
interna entre las instituciones de los diferentes niveles considerando que el paso de un nivel al otro no debe entenderse como una barrera sino en función de las trayectorias educativas.

La historia de nuestra educación da cuentas de una cuestión estructural muy desigual donde el acceso a la educación, sobre todo el nivel superior, se vuelve selectivo y suele no formar parte del abanico de opciones de centenares de jóvenes a la hora de pensar su futuro, sobre todo de quienes provienen de contextos socioculturales adversos. Si nos centramos en la obligatoriedad de la escuela se ponen en tensión las trayectorias ideales/teóricas con las trayectorias reales de los estudiantes (Terigi, 2011). Las representaciones acerca de lo que un estudiante de determinado nivel debería ya saber y saber hacer, esto es, las representaciones acerca de un oficio que se considera debiera ya estar plenamente aprendido, entran en discusión con los desempeños académicos de los sujetos sociales que ingresan a las aulas, y especialmente, con aquellos que históricamente estuvieron excluidos.

En el caso del nivel superior, tiene como particularidad que no reviste condición de obligatoriedad; sin embargo, la Ley de Educación Nacional sostiene en su artículo 11: «b) Garantizar una educación integral que desarrolle todas las dimensiones de la persona y habilite tanto para el desempeño social y laboral, como para el acceso a estudios superiores». Es decir, ingresar a estudios superiores es una elección personal que suele estar teñida por diferentes circunstancias de distinta índole y que requiere no perder de vista la complejidad de la transición hacia la vida adulta a partir de la continuidad o no de estudios superiores. Esta transición posibilita delinear un camino abierto que, más allá del circuito institucional propuesto, los sujetos pueden transitar y desde el cual construyen múltiples significados.

Los problemas de articulación entre las escuelas secundarias y la universidad son de naturaleza compleja. Las acciones con el último año del nivel secundario cobran relevancia en tanto constituyen momentos de decisión respecto a proyectos de vida de muchos jóvenes que han finalizado la educación secundaria. El trabajo con estudiantes y docentes resulta trascendental, a los efectos de poner en valor la continuidad de los estudios superiores, y procurar la reflexión crítica acerca del papel que el conocimiento científico desempeña con respecto al progreso de la humanidad y las posibilidades que trae aparejada la continuidad 
de los estudios superiores. Considerar ésta como un universo posible permite ampliar las perspectivas profesionales futuras y promover transformaciones valiosas en vistas a lograr la igualdad de oportunidades (Pacífico, 2013).

En este artículo nos proponemos compartir la experiencia llevada adelante en el proyecto Nexos como una estrategia innovadora, que recupera el aula como un espacio vital de enseñanza, intercambio, promoción de derechos, y encuentro entre ambos niveles.

\section{Educación Superior. Experiencia Nexos}

El programa Nexos es una política educativa de integración entre los distintos niveles y jurisdicciones del sistema educativo, que tiene el objetivo de generar condiciones institucionales y curriculares que posibiliten procesos genuinos de inclusión y permanencia en los estudios universitarios, a través del trabajo conjunto entre docentes y estudiantes de ambos niveles. El proyecto se lleva a cabo mediante un trabajo articulado entre el Ministerio de Educación de la Provincia de Santa Fe y la Universidad Nacional del Litoral, y es financiado por la Secretaría de Políticas Universitarias del Ministerio de Educación de la Nación. El proyecto planteó cuatro líneas de trabajo que se llevaron a cabo en escuelas de localidades de las provincias de Santa Fe, Entre Ríos y Córdoba. En Tutorías en la Escuela Secundaria se propuso promover y orientar la continuidad de estudios superiores en estudiantes de los últimos años. En este sentido, se desarrollaron las siguientes actividades: a) Tu escuela en la universidad: buscó el acercamiento de los futuros ingresantes a la institución universitaria mediante recorridos por lugares emblemáticos y estratégicos de las diferentes unidades académicas; b) La universidad en tu escuela: es un espacio interinstitucional que fomenta el diálogo entre estudiantes de ambos niveles con el fin de pensar y problematizar sus ideas en torno a continuar estudios superiores, c) La UNL en tu ciudad: mediante visitas a localidades del interior de las provincias de Santa Fe, Entre Ríos y Córdoba se buscó acercar la propuesta académica, participando en ferias de carreras y talleres de orientación vocacional. En la línea Innovación educativa: producción de material educativo se buscó contribuir 
en los procesos de construcción de los proyectos de vida de los jóvenes que egresan de la educación secundaria.

A partir del material del Curso de Articulación General Ciencia, Arte y Conocimiento (CAC) de la UNL, que propone un recorrido por diferentes tipos de textos (escritos, fílmicos, pictóricos), se diseñaron diversas actividades con el fin de acercar al estudiantado a una problemática histórica y a la vez nueva: la interacción entre las ciencias, las tecnologías y las artes en la producción de los conocimientos, generando materiales innovadores.

Esta propuesta se llevó a cabo en 15 escuelas ubicadas en las localidades de Santa Fe, Reconquista, Vera, Gálvez, Esperanza y Santo Tomé. Para ello, fueron necesarios numerosos encuentros con áreas de gestión del Ministerio de Educación de la Provincia de Santa Fe, equipos directivos de las escuelas secundarias, docentes y tutores universitarios del curso CAC, y docentes designados por las escuelas, con el fin de socializar los objetivos del proyecto y evaluar las condiciones institucionales para su implementación. En relación a la propuesta, se acordó considerar aprobado el curso de articulación general CAC a aquellos estudiantes que cumplieran con los requisitos de aprobación. Se conformaron 31 comisiones de trabajo, integradas por docentes de nivel secundario y tutores docentes de la UNL, teniendo en cuenta para su implementación las particularidades de cada grupo, división e institución. Participaron más de 800 jóvenes del último año de las escuelas, más de 450 fueron evaluados satisfactoriamente, de los cuales 333 registraron su inscripción en alguna carrera de la UNL. Por otro lado, en la línea acompañamiento de las trayectorias educativas se hizo foco en construir criterios comunes de enseñanza, aprendizaje y evaluación de contenidos nodales disciplinares en el espacio de articulación de niveles y elaborar conjuntamente una propuesta de enseñanza en el último año del nivel. Para ello, se conformaron equipos mixtos que desarrollaron sus acciones en 19 escuelas ubicadas en Santa Fe, Reconquista, Gálvez, Esperanza y Santo Tomé. Con el fin de presentar la propuesta y metodología de trabajo, se realizaron diversos encuentros con áreas de gestión del Ministerio de Educación y directivos de las escuelas. Posteriormente se realizaron encuentros con docentes de ambos niveles, pertenecientes a las áreas de conocimiento elegidas para el abordaje áulico: Matemática, Ciencias Sociales, Ciencias Naturales (química y biología); Contabilidad; Lengua (lectura y escritura de textos académicos). Se 
conformaron 47 comisiones de áreas disciplinares: 21 de Matemática; 6 de Lengua; 7 de Ciencias Sociales; 10 de Ciencias Naturales; y 3 de Contabilidad. En encuentros semanales, los estudiantes trabajaron contenidos acordados por los docentes. Estos fueron propicios para la habilitación de espacios de reflexión sobre las propias prácticas, habituales o rutinarias, que tienen lugar en la vida cotidiana de las escuelas. Por último, en la línea de Estrategias de formación y capacitación permanente se trabajó en el marco del Programa de Formación Permanente Escuela Abierta y se diseñó en forma conjunta con el Ministerio de Educación de la Provincia una propuesta de formación en todos los niveles del sistema educativo para problematizar sobre los sentidos de la articulación de niveles. La intención fue identificar obstáculos, quiebres, continuidades, encuentros y desencuentros que gravitan en las diferentes trayectorias educativas, facilitando u obturando el acceso, permanencia y egreso a los niveles inmediatos superiores. En tal sentido, se enviaron un conjunto de materiales para ser abordados por directivos, docentes y estudiantes que invitaban a reflexionar en torno a las experiencias vividas en el tránsito entre niveles.

Al finalizar la jornada se completó, de forma anónima, una encuesta que pretendió recuperar las diferentes perspectivas y miradas de quienes integran el sistema educativo sobre la problemática de la articulación de niveles. La misma fue respondida por un total de 24.281 personas, quienes reflexionaron en torno a las siguientes dimensiones de análisis, a saber: valoración de la experiencia; percepciones sobre la educación; aprendizajes de cada nivel; prácticas de articulación e imaginarios sobre la transición entre niveles.

La primera dimensión fue valorada positivamente por equipos directivos, docentes y estudiantes de todos los niveles y modalidades. Se destacó la participación de estudiantes que, al reconstruir sus trayectorias, permitieron la emergencia del rico lenguaje de las emociones. Asimismo, recuperaron: el disfrute por la participación de una actividad atípica en la escuela; el gusto por recordar momentos pasados en el espacio escolar; el compartir con compañeros y alumnos de otros cursos; y el ejercicio reflexivo de auto-observación del tránsito escolar sumado a una proyección a futuro en base a sus deseos y expectativas de vida.

Las percepciones respecto a la educación se muestran coincidentes entre docentes y alumnos: el sentido de dar y recibir educación está dado por la formación en valores (ciudadanía, responsabilidad, ejercicio de la libertad, autonomía, 
tolerancia); la construcción de una identidad positiva (digna), así como también proporcionar herramientas más prácticas para insertarse socialmente en un mundo complejo. Los docentes destacan la idea de recuperar la pasión y los sentidos de educar. El deseo de formar ciudadanos transformadores, constructores de sociedades más igualitarias también está presente en las escuelas. Por su parte, los estudiantes perciben la educación representada con ideas más abstractas (progresar, desarrollarse) o ideas más concretas (conseguir un trabajo). Asocian la educación a expectativas con respecto al futuro y a ciertos «beneficios»cuyos frutos se verán «más adelante»: la imaginación de un futuro promisorio; construir una identidad que les permita posicionarse frente a otros; aprender herramientas específicas para desenvolverse; disfrutar de una vida digna; ser alguien en la vida y aprender valores para la convivencia en sociedad. Respecto a las últimas dos dimensiones, de las respuestas surgen que los protagonistas vivencian las transiciones como procesos complejos, cargados de incertidumbre, espacios indeterminados, territorios difusos, sin determinaciones claras. Los alumnos se muestran críticos y preocupados respecto a la preparación recibida para enfrentar el nivel siguiente, donde hay expectativas y también temores. Los docentes reconocen este objeto de trabajo: lo piensan, lo asumen, proponen actividades, señalan los distintos aspectos involucrados. Sin embargo, también aparece como una fuente de frustración; de resultados y logros que no se alcanzan (Blesio, Pacífico y Trevignani; 2019).

\section{Consideraciones finales}

La creación del programa Nexos de la SPU, implementado a través de los Consejos Regionales de Planificación de la Educación Superior (CEPRES), constituye un dispositivo muy eficaz en el marco de las políticas de articulación. En el caso de nuestra Universidad, se vienen implementando hace más de dos décadas diferentes líneas de trabajo que proponen estrategias de vinculación con estudiantes e instituciones educativas del nivel secundario.

Para poner en palabras la experiencia compartida, lo primero que quisiéramos destacar es que para implementar Nexos fue necesaria la predisposición y voluntad 
política de autoridades y docentes de ambas jurisdicciones, que nos permitieron iniciar un trabajo conjunto y comprometido fomentando un diálogo fecundo. Las actividades realizadas en el marco del Proyecto Nexos constituyeron una experiencia innovadora en lo que respecta a la articulación entre la escuela secundaria y la Universidad., generando espacios de encuentros y trabajo conjunto. Las diferentes líneas de trabajo que sostuvieron este proyecto dan cuenta de políticas pensadas para promover el diálogo fecundo entre ambos niveles, cuyos principales protagonistas fueron cientos de jóvenes estudiantes que transitaban los últimos años de la educación obligatoria, quienes pudieron comunicarse e interactuar con estudiantes universitarios para informarse, diluir dudas y miedos comunes.

Por un lado, la habilitación del aula escolar para el trabajo conjunto y colaborativo entre docentes, donde la escucha, la empatía y el reconocimiento de las dificultades que cada escuela debe afrontar cotidianamente, se convirtió en una oportunidad para revisar prácticas educativas que hoy tienen vigencia en el nivel superior, teniendo en cuenta las peculiaridades de cada establecimiento educativo.

Por otro lado, es de destacar la oportunidad brindada por el Ministerio de Educación de la Provincia de Santa Fe en el marco del programa de formación permanente "Escuela Abierta», que nos permitió llegar a toda la comunidad educativa del territorio provincial, abriendo el debate sobre la temática de articulación de niveles y cuyos resultados obtenidos a partir de la encuesta fueron sistematizados en un informe digital editado por el Centro de Ediciones UNL, disponible en la web para todas las escuelas. En tal sentido, vale aclarar que aún queda pendiente un trabajo conjunto de análisis de datos y conclusiones entre ambas jurisdicciones en pos de seguir fortaleciendo los procesos de articulación ya iniciados.

A modo de cierre, Ana María Ezcurra (2013) sostiene que «la enseñanza es un condicionante primario, en cualquier tipo de alumnos, pero sobre todo en población de estatus en desventaja». Por ello, entendemos que esta experiencia de trabajo concretada en el marco del proyecto Nexos constituye un puntapié inicial de un camino a consolidar y fortalecer, en donde se recuperan y respetan las particularidades de cada institución educativa, así como también se generan y fortalecen los espacios de escucha a los jóvenes y entre docentes. 


\section{Referencias bibliográficas}

Arendt, H. (1993). Entre el pasado y el futuro, ocho ejercicios sobre la reflexión política. Barcelona: Herder. Blesio, M.; Pacífico, A.; Trevignani, V. (2019). Sentidos, sentires y prácticas en el sistema educativo santafesino. Santa Fe: Universidad Nacional del Litoral. Ezcurra A. M. (2013). Igualdad en educación superior. Un desafío mundial. Los Polvorines, Prov. de Buenos Aires. Universidad Nacional de General Sarmiento e Instituto de Estudios y Capacitación Federación Nacional de Docentes Universitarios.

Pacífico A. et al. (2013). Análisis del acceso a la UNL, los dispositivos político-académicos y la normativa institucional como herramienta para la igualdad de oportunidades. Artículo presentado en $V$ Encuentro Nacional y II Latinoamericano sobre Ingreso a la Universidad Pública "Políticas y estrategias para la inclusión. Nuevas complejidades; nuevas respuestas». Luján.

Pacífico, A y Saccone, J. (Comps.) (2016). Tutorías entre pares en la Universidad Nacional del Litoral. Alcances y desafíos. Santa Fe: Ediciones Universidad Nacional del Litoral.
Rascován, S. (2016). La orientación vocacional como experiencia subjetivante. Buenos Aires: Paidós.

Terigi, F. (2007). Los desafíos que plantean las trayectorias escolares. Fundación Santillana III Foro Latinoamericano de Educación Jóvenes y docentes. La escuela secundaria en el mundo de hoy. 28, 29 y 30 de mayo de 2007.

Terigi, F. (2011). Las cronologías de aprendizaje: un concepto para pensar las Trayectorias escolares en Pensar la escuela 2. Ministerio de Educación de la Nación. Buenos Aires.

Universidad Nacional del Litoral (2016). Ingresantes a la Universidad Nacional del Litoral 2010-2014. Hacia una mayor inclusión educativa.

\section{Documentos consultados:}

Declaración de la CRES. Universidad Nacional de Córdoba, 2018

Ley de Educación Nacional № 26.206 (2006). 